\title{
Attempt TCP ACK-Storm Based Virtual Network Attacks and Defence Solutions
}

\section{Tran Namm Khanh}

Dai hoc Ky thuat Le Quy Don

Ta Minh Thanh ( $\nabla$ taminhjp@gmail.com )

Dai hoc Ky thuat Le Quy Don https://orcid.org/0000-0001-8327-2009

\section{Research Article}

Keywords: DoS, Hypervisor, TCP, ACK storm, Virtual network, Docker network.

Posted Date: January 6th, 2022

DOI: https://doi.org/10.21203/rs.3.rs-420162/v1

License: (c) (i) This work is licensed under a Creative Commons Attribution 4.0 International License. Read Full License 


\title{
Attempt TCP ACK-Storm based Virtual Network Attacks and Defence Solutions
}

\author{
Tran Nam Khanh ${ }^{\mathrm{a}, 1}$, Ta Minh Thanh ${ }^{\mathrm{b}, 1, \star}$ \\ ${ }^{1}$ Le Quy Don Technical University, 236 Hoang Quoc Viet, Ha Noi, Viet Nam
}

Received: date / Accepted: date

\begin{abstract}
The rapid development of the digital age has been pushing people to access a mobile working environment when handsets are becoming more diverse and convenient with the help of Virtualization Technology. The speed and usability of Virtualization Technology are astounding for saving initial investment costs and optimizing IT infrastructure. Such Virtualization Technology is what businesses are interested in and makes the virtual server market growing strongly, especially for businesses that have many branches. However, virtual systems (hypervisors) are more vulnerable than traditional servers according due to many network attacks from curious users. Therefore, it's necessary to prepare for the worst circumstances, understand clearly, and research for new threats that can break down the virtual system. In this paper, we attempt to demonstrate the TCP ACK storm based DoS (Denial of Service) attack on virtual and Docker networks to show the threats that easily are happen on services deploying on virtual networks. Based on such consequence, we propose some solutions to prevent our virtual system from potential risks.
\end{abstract}

Keywords DoS · Hypervisor · TCP · ACK storm · Virtual network $\cdot$ Docker network.

\section{Introduction}

\subsection{Overview}

\subsubsection{Age of Server Virtualization}

According to the recent reports, server virtualization is becoming more and more popular and widely used. In November 2012, Jake McTigue mentioned in the "2013 Virtualiza-

\footnotetext{
${ }^{\star}$ Corresponding author

ae-mail: noange10607@gmail.com

be-mail: thanhtm@mta.edu.vn
}

tion Management Survey" [1] of "Information Week" that 42 percents of 320 business technology respondents said their companies use multiple hypervisors, and the usage would be 87 percent in 2013. After that, Spiceworks conducted a survey [2] of 539 IT decision-makers of businesses with variety of company sizes, including small businesses, midsize businesses and enterprises, and variety of industries, including education, healthcare, nonprofits, government, finance, retail, construction, manufacturing, and IT services, in September 2019. The survey of Spiceworks points out that most businesses use server virtualization, with 92 percents using currently and 5 percents planning to use within 2 years. For businesses, it is important to achieve maximum efficiency with minimum costs, and optimal costs for IT infrastructure is also one of those achievements. Virtualization technology, especially server virtualization, has been doing very well. For providing web hosting services and effectively utilizing existing resources in IT infrastructure in a cost-effective way, we can use server virtualization. Before virtualization servers, servers used only a fraction of their processing power, and work less effectively, because only a portion of the network's servers handle the workload. This results in data centers become overcrowded with underutilized servers, causing a waste of resources and power. To overcome this situation, server virtualization divides each physical server into multiple virtual servers, and allows each ones to act as a unique physical device such as run its own applications and operating system. By this way, server virtualization provides many benefits: higher server ability, making operating costs cheaper, eliminating server complexity, increasing application performance and deploying workload quicker. 


\subsubsection{Common Types of Server Virtualization}

There are 3 popular types of virtual servers for businesses to choose from: Full virtualization, Para-virtualization and OS-level virtualization [4].

With full virtualization, a hypervisor, which is a type of software serving as a platform for the virtual servers' operating systems, interacts with a physical server's disk space and CPU directly. It monitors and relays the physical server's resources to the correct virtual server as it runs applications. In other words, it provides necessary everything for virtual server, and responds to privileged CPU instructions and handles queuing, dispatching and returning the hardware requests. The hypervisor also keeps each virtual server independent and unaware of the other virtual servers. However, the speed of the application and server performance can be be reduced because a hypervisor has its own processing needs.

Para-virtualization approach with a bit different way, the guest servers in a para-virtualization system are aware of one another. This results in it involves the entire network working together as a cohesive unit. It use a hypervisor too, but it doesn't need as much processing power to manage the virtual servers, because each one is already aware of the demands the others are placing on the physical server.

Unlike the previous two, a hypervisor wasn't include in OS-level visualization. Instead, the virtualization capability, which is part of the physical server operating system, performs all the tasks of a hypervisor. The biggest limitation of this approach is that all guest servers must run on the same operating system. Each virtual server remains independent of all others, but it cannot use these servers, different operating systems and harmonize them. Since all the guest operating system should be the same, such an environment is called homogeneous.

\subsubsection{Challenges of Server Virtualization}

Although the emergence of server virtualization offers many benefits in both efficiency improvements and lower infrastructure costs, it still has many security flaws that may effect on real services. There are many potential cyber security threats to a hypervisor system. In the research scope of the paper, we focus on overcoming some of the following network security problems:

1. The DoS/DDoS attack is one of many threats. In 2019, Imperva [3] had reported an SYN DDoS attack in which 500 million packets per second (PPS) in January and another in which 580 million packets per second (PPS) in April. Each of the packets was thought to a median number of 850 bytes per packet. That means that 580 million 850-byte packets would result in about 3944 Gbps of data targeting your network protocol every second to render it unresponsive. Previously, in 2018, the GitHub DDoS Attack [3] was recognized as sustaining a 1.35 Tbps (with 129.6 million PPS) attacks without the help of botnet. "Size" of DoS/DDoS attacks is increasing year by year and cost businesses thousands to millions of dollars in losses. To prevent and minimize the DoS/DDoS attack's sabotage, analyzing more types of DoS attack is necessary, especially with server virtualized environment.

2. If attackers combine the ACK-Storm DoS attack with a botnet, they could make virtual server fall into a state of port-exhaustion in a period of time. That affects the real services cannot response the requests from users. In the real applications, users [5] will leave your applications if there are problems on response time and system performance. Therefore, detecting the network attacks on virtual network (even that happens in a period of time) to prevent it is quite important for real applications such as web-based, API-based applications.

3. After detecting the network attacks on the virtual system, how to prevent the attacks and to avoid the risk, is also important challenging issue. Therefore, we also consider to propose the solutions for improving the security of virtual networks.

\subsection{Our contributions}

In our knowledge, the research on the attacks to the vSwitch/vBridge of hypervisor systems and docker systems is not focused on, especially on the docker. Therefore, real services deployed on hypervisor systems or docker systems are vulnerable to attack via a network. That is the motivation of our paper to research the related network attacks on such systems. In summary, we briefly introduce our contributions in this paper as follows:

1. We propose to attempt DoS attacks using FIN-ACK storm on services deployed on virtual systems so that service providers understand the risks, and security vulnerabilities when deploying the services in a virtual environment. That implies that real applications deployed on virtual systems can be easily attacked by hackers via the Internet.

2. The DoS attacks proposed on the Virtual Machine and Docker systems in this paper is the first attempt that is made to prove feasible when a hacker wants to attack services on a hypervisor system.

3. We propose a new attack method based on ACK-Storm DoS attack with FIN-ACK packet which can make vSwitch/ vBridge fall into a state of port-exhaustion in a period of time. 
4. Based on these attack experiments, our paper also offers some solutions to prevent and decrease the destruction of these types of attacks in real applications.

\subsection{Roadmap}

The rest of this paper is organized as follows: In Section 2, a brief overview of the related works are presented. We focus on the explanation of the ACK-Storm DoS attack and the FIN-ACK-Storm DoS attack. To illustrate, in Section 3, the detail of the proposed DoS attacks on the hypervisor systems, VMware and Docker, are explained. Based on our experimental attacks, we show the simulation results and discussion about the feasibility of the Ack-Storm DoS attack on hypervisor systems. Furthermore, Section 4 describes in detail some proposed solutions, some experimentation, and evaluation of those measures. Finally, Section 5 gives the conclusions of this paper.

\section{Related works}

\subsection{Related techniques}

\subsubsection{Three-step handshake}

Three-step handshake, or maybe called a Three-way handshake, is used in TCP to establish a connection. TCP uses passive open, a server bind to and listens to a port before a client tries to connect to the server. A client may start an active open after the passive open is established. The threestep handshake occurring in three steps (see Fig 1) can be described as follows:

1. The client, who wants to connect to the server, sends a TCP packet to the server with a random value A for the segment's sequence number (SEQ) and a bit SYN set. This packet is called a SYN packet (1).

2. After the server receives the $S Y N$ packet, it responds to the client a TCP packet with a random value B for SEQ number, the acknowledgment number is set to one more than the received sequence number $(\mathrm{A}+1)$, and two-bit SYN, ACK are set. This packet is called a SYN-ACK packet (2).

3. Finally, the client sends an ACK packet (3), which has the acknowledgment number is set to one more than the received sequence number $(B+1)$, the sequence number is set to the received acknowledgment value $(\mathrm{A}+1)$, and only bit ACK set.

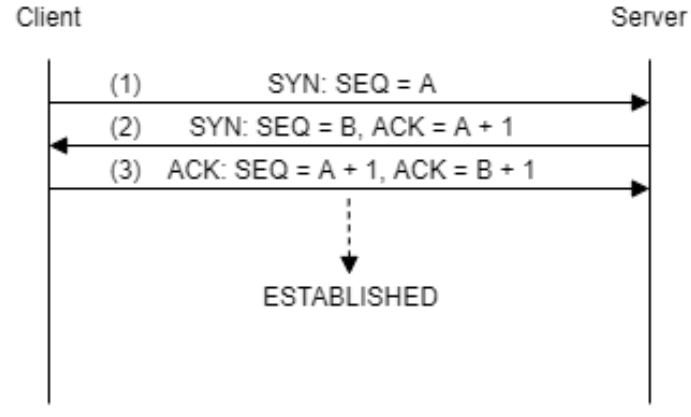

Fig. 1 TCP Three-step Handshake - Establish TCP connection

\subsubsection{Four-step handshake}

Four-step handshake, or four-way handshake, is used to terminate TCP connection with each side of the connection terminating independently. It occurs as follows:

1. When one party X of a TCP connection wants to terminate its half of the connection, it sends to the other endpoint a FIN packet, which has FIN bit set, the SEQ number (A), and ACK number (B) depending on the current state of the TCP connection. Then, it enters the FINWAIT-1 state.

2. The other endpoint $\mathrm{Y}$ receives the FIN packet, free up its buffer, and responds an ACK packet, with acknowledgment number is more one than the received sequence number $(\mathrm{A}+1)$ and the sequence number is set to the received acknowledgment value (B). It enters the CLOSEWAIT state. After receiving the ACK packet from endpoint Y, endpoint X enters the FIN-WAIT-2 state from the FIN-WAIT-1 state. The connection from endpoint $X$ to endpoint $\mathrm{Y}$ is terminate, but the connection from endpoint $\mathrm{Y}$ to endpoint $\mathrm{X}$ still opens, this is the half-close connection.

3. Endpoint $\mathrm{Y}$ sends a FIN packet to terminate the connection from endpoint $\mathrm{Y}$ to endpoint $\mathrm{X}$ and waits for an acknowledgment from endpoint $\mathrm{X}$.

4. Finally, when endpoint $X$ receives the FIN packet from endpoint $\mathrm{Y}$, it enters the CLOSED state.

\subsubsection{VMware Workstation}

Virtual Machines (VMs) are software computers that provide the same functionality as physical computers. They are based on computer architectures but they behave as separate computer systems. With them, the users could run different software requesting different environments without conflict at the same time. They bring many benefits for users and businesses: Reduced hardware costs; Faster desktop and server provisioning and deployment; Small footprint and energy saving; Increasing IT operational efficiency, and so on. 


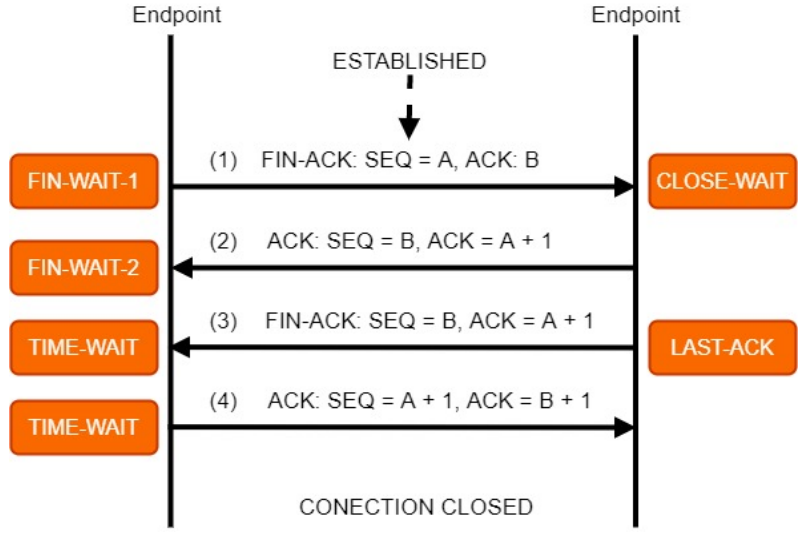

Fig. 2 TCP Four-step Handshake - Terminate TCP connection

Hypervisor, or a virtual machine monitor (VMM), can be hardware, software, or firmware that provides virtualization capability. A hypervisor allows one host computer, which the hypervisor operates in, to support multiple guest VMs by virtually sharing its resources such as memory and processing, and so on. According to the resources that have been allocated for each virtual machine, the hypervisor gives and manages the scheduling of VM resources against the physical resources. The hypervisor has two types: type 1,"bare metal", run directly on the host's hardware, like an operation system, while type 2, "hosted", run as software on an operating system, as an application. What type of usage is based on the purpose and need of the user and businesses.

Virtual Switch (vSwitch) is a software application that allows communication between virtual machines, between the physical machine and virtual machines. It directs the communication on a network in an intelligent way by ensuring the integrity of the virtual machine's profile, which includes network and security settings checking data packets before moving them to a destination. A virtual switch is completely virtual and can connect to a network interface card (NIC). The vSwitch merges physical switches into a single logical switch. This helps to increase bandwidth and create an active mesh between a server and switches. It also helps in easy deployment and migration of virtual servers, allows network administrators to manage virtual switch deployed through a hypervisor, and easy to roll out new functionality, which can be hardware or firmware related.

Virtual network - Network Virtualization is a method of splitting up the available bandwidth into channels to combine available resources in a network. Each of the channels can be assigned (or reassigned) to a particular server or device in real-time and is independently secured. The main idea of Network virtualization is that virtualization disguises the true complexity of the network by splitting it into manageable parts, like a partitioned hard drive, making it easier to manage files. Every subscriber has shared access to all the resources on the network from a single computer.

\subsubsection{Docker}

Docker Engine, which may be called Docker Daemon, is a background-running service that manages everything required to run and interact with Docker containers on the host operating system. It's used to run Docker containers which bundled up all application dependencies inside. Docker Engine enables containerized applications to run anywhere consistently on any infrastructure. Docker Daemon communicates directly with the host operating system and knows how to ration out resources for the running Docker containers. It's also an expert at ensuring each container is isolated from both the host OS and other containers. In simple terms, it replaces the hypervisor.

Docker container image, or Docker image, is a lightweight, standalone, executable package of software. It includes code, runtime, system tools, system libraries, and setting files - all things needed to run an application. There are many Docker images available that could be used to rebuild new images or deployed Docker containers.

Docker Container is an instance of a deploying Docker image. But, we could modify Docker containers. Multiple containers can run on the same machine at the same time and share the OS kernel. They run as independent processes in userspace. Containers take up less space than VMs, can handle more applications, and require fewer VMs and Operating systems. Docker Network: the Docker networking philosophy is application-driven. Docker network isolation achieved using Network namespace. Typically, services gets separate IP and maps to multiple containers. Microservices done as Container puts more emphasis on integrated Service discovery. As the Container scale on a single host can run to hundreds, host networking has to be very scalable. Virtual Bridge (vBridge), which may be called Network bridge or Linux bridge, is a piece of software used to unite two or more network segments. It works like a virtual network switch (vSwitch) and working transparently [18] [19]. In Docker container built with a Linux image base, the Docker network is managed by vBridge.

\subsubsection{Network (NAT) And Port Address Translation (PAT)}

Network Address Translation (NAT) is a technique that allows one or more internal IP addresses to be converted to one or more external IP addresses. This technique makes a device in a local/private network could connect to the public network (Internet). NAT is responsible for transmitting packets from one network layer to another in the same network. NAT will make changes to the IP address inside the packet, then move through routers and network devices. On the contrary, when the packet is transmitted from the internet (public) back to the NAT, NAT performs the task of changing the destination address to the IP address inside the local 
network and sending it. Moreover, NAT can act as a firewall. It helps users secure computer IP information. Specifically, if the computer is having trouble connecting to the internet, the public IP address (previously configured) is displayed instead of the local network IP address.

Port Address Translation (PAT) is an extension technique of NAT which could help multiple devices on a local/private network connect to the public network by mapping their local IP address to a single public IP address and specific ports. With each set of local-IP:local-port is mapped to a public IP address with a specific port, multiple devices could communicate with the Internet with the corresponding ports provided to them. This technique could conserve IP addresses but the number of ports is not unlimited, only 65,536 ports so there can be a theoretical maximum of 65536 PAT entries at a time for each inside global address. If an attacker can occupy all 65,536 ports, there is a port-exhaustion, and no communications can be made between local devices and the public network.

\subsection{Related attack techniques}

\subsubsection{Man-in-the-middle - MITM}

MITM is a term for a cyberattack technique [20] in which an attacker places himself between the server and client connection (or between clients) (see Fig. 3), or pretends to be the other party in a connection. This MITM attack tries to steal sensitive information such as login certifications, social network accounts or password of bank accounts, to modify correspondences between them. The targets of MITM are generally financial applications, SaaS businesses, webbased business and other sites. By using MITM network attack technique, criminals can obtain a lot of sensitive information to serve bad purposes, causing financial damage to individuals and businesses. This network attack technique is also used as a stepping stone to other more advanced attack technique. Attackers can take advantage of the ability to eavesdrop on network communication messages between victims and insert malicious messages into that conversation.

Furthermore, in dynamic eavesdropping, the attacker creates fake connections between the victims/clients by creating independent connections with the victims/clients and transferring messages between them to make them to trust they are talking directly to each other over a private connection. The attacker must be able to intercept all significant messages passing between the two casualties and inject new ones. For example, with an unencrypted wireless access point (WiFi) [21] [22], the attacker could use MITM attack to eavesdrop messages between clients in the unencrypted $\mathrm{WiFi}$, between clients and services in the Internet.

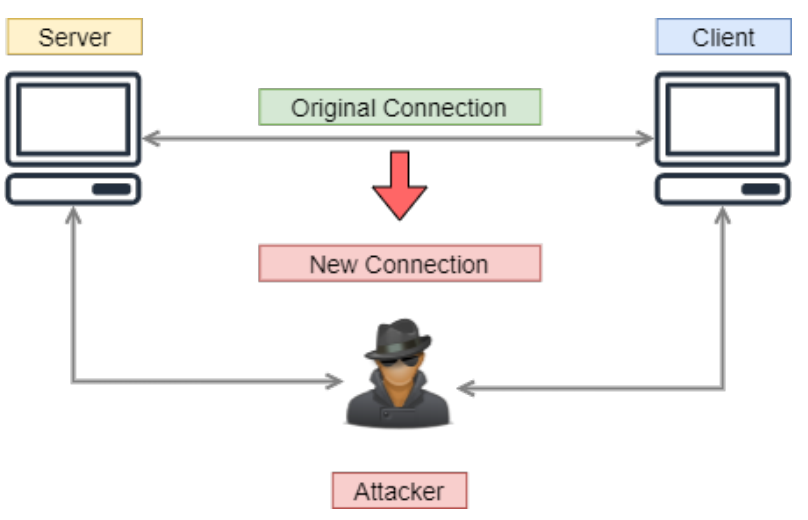

Fig. 3 Man-in-the-middle attack model

Such MITM attack also can be used for attacking the virtual services in order to enable the DoS/DDoS attacks. This paper proves that MITM attacks is effectively employed to achieve the information feeding in the DoS/DDoS attacks.

\subsubsection{Original ACK-Storm DoS attack}

The original idea [6] is suggested by Abramov and Herzberg depending on the vulnerable handle exceptions of TCP that described on page 72 of RFC 793 [12] about TCP: when a TCP connection is in the ESTABLISHED state received a packet with not-yet-sent acknowledges data SEG.ACK > SND.NXT (Acknowledgement from the receiving TCP higher than the sequence number of the next byte of data to be sent to the other), the received one handle as follows: Send an ACK (with the last sent SEQ/ACK number) to another party of connection, then drop the segment and return. In particular, ignore the payload in the segment. However, this state has a timeout and stopped when the timer reaches the timeout. For raising the basic ACK-storm DoS attack (Two Packets ACK Storm), the attackers act as the following scenario:

1. Pick up (at least) one packet from a TCP connection between a client and a server (Just need to eavesdrop one packet and do not need any impacts on the connection).

2. Generate two packets, each addressed to one party, and with a sender address of the other party (i.e. spoofed). The packets must be inside the TCP windows of both sides. The packets should have content - at least one byte of data or it will not be implemented.

3. Send the packets to the client and the server at the same time. The connection will then enter an infinite loop of sending ACK packets back and forth between both parties.

\subsubsection{ACK-Storm DoS attack using FIN-ACK packet}

Depending on Abramov's idea [6], Son proposed another ACK-Storm DoS attack [7] with the same mechanism, but 


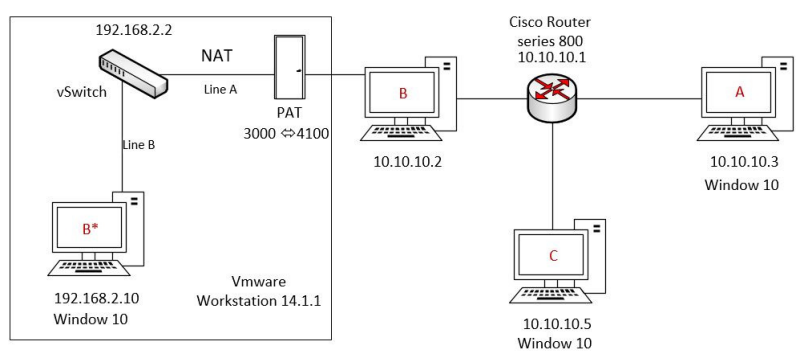

Fig. 4 Experimental model - Three physical computers as above connected with each other by a router Cisco 800 Series Routers CISCO881-K9, the $\mathrm{C}$ is attacker which can eavesdrop and inject packets into TCP connection between Host A and Hypevisor B

the starting point is sparked by a couple of FIN-ACK packets created by the same way when creating a couple of ACK packets to trigger ACK-Storm DoS attack. According to the description in RFC-793 [12], if a TCP connection is in CLOSEWAIT state, it does the same processing as for the ESTABLISHED state. That means if attackers force each party running into CLOSE-WAIT, each party waits for an ACK packet (see Fig. 2) never come but not-yet-sent ACK packet instead, then the ACK-Storm DoS raised by two FIN-ACK packets starts. Because no timeout by default for CLOSE-WAIT state, this DoS attack will never stop in theory, and parties of the connection will stay in CLOSE-WAIT state forever.

\section{Attack experiments on virtual systems}

\subsection{Experimental environment}

Physical Computers Host A and Hypervisor B have the same

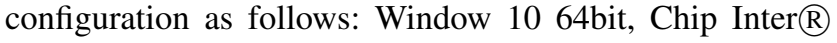
Core $^{\mathrm{TM}}$ i7-6700 CPU @ 3.40GHz, RAM 8GB, the network interface is a $100 \mathrm{Mbps}$ Ethernet adapter attached to the PCIE bus. The attacker $\mathrm{C}$ is Windows 10 installed Python.

Router We use a router instead of the hub in Son's experimental test [7] because, in reality, businesses use the router for establishing their LAN network or connect to the Internet. The router that is used in our experiments, is a Cisco 800 Series Routers CISCO881-K9.

Our experimental environment is shown in Fig. 4, and Fig. 5. This experiment shows the concept of the virtual machine system (Hypevisor B) installed inside physical computer. We capture packets simultaneously between server B and vSwitch/vBridge - Line A, between vSwitch/vBridge and server $\mathrm{B}^{*}$, Line $\mathrm{B}$. Based on the captured packets, we verify the feasibility of the attack technique, the feature of

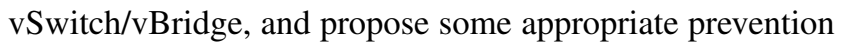
measures.

\subsection{Experiments on VMWare Workstation}

Hypervisor: We use VMware Workstation 14.1.1 installed Windows 10 64bit for Virtual Machine server B* in both experiments on VMWare Workstation and Docker.

\subsubsection{Original ACK-Storm DoS attack on Virtual Machine}

Attack Execution: We use a simple TCP connection created with socket python (v3.7.3) scripts. For the experiment, we just let $A$ and $B^{*}$ create a connection with a Three-Way Handshake and pick up the last ACK packet from the connection for SEQ and ACK sequence number. Then, in attacker $\mathrm{C}$, we use scapy ${ }^{1}$ to create a couple of fake ACK packets with source IP is one party and destination IP is the other. We sent those ACK packets to each of the respective parties.

Analysis: When each party received fake ACK packets, they respond with invalid retransmitted ACK packets, and the ACK-Storm was begun.

In Line A: We captured about 55000 retransmitted ACK packets in 60 seconds while the ACK storm was occurring. This result is similar to the results in previous experiments of Abramov [6] and Son [7] but the number of packets is less because of smaller Ethernet adapter bandwidth. However, task manager still displayed the bandwidth used by VMware NAT services was $0.6 \mathrm{Mbps}$. This result is much bigger than 120 bytes (two packets) sent by the attacker $\mathrm{C}$.

In Line B: Only the first fake ACK packet, which we created, is forwarded to virtual machine $\mathrm{B}^{*}$ through vSwitch. No retransmitted ACK packet is directed to virtual machine $\mathrm{B}^{*}$.

\subsubsection{ACK-Storm DoS attack using FIN-ACK packet}

Attack Execution: We do the same action with the experiment ACK-Storm DoS attack but we use a couple FIN-ACK packets instead of a couple of ACK packets. It means that we just turn the bit FIN flag to "1" and keep all conditions as the experiment ACK-Storm DoS attack as above.

Analysis: When each party received fake FIN-ACK packets, they respond with invalid retransmitted ACK packets, and the ACK-Storm was begun.

In Line A: We captured about 86000 ACK retransmitted packets in 60 seconds (about 1434 packets per second) in Line A while the ACK storm was occurring. This result is similar to the results in previous experiments of Abramov [6] and Son [7] but the number of packets is less because of smaller Ethernet adapter bandwidth. However, the task manager still displayed the bandwidth used by VMware NAT

\footnotetext{
${ }^{1}$ https://scapy.net/
} 


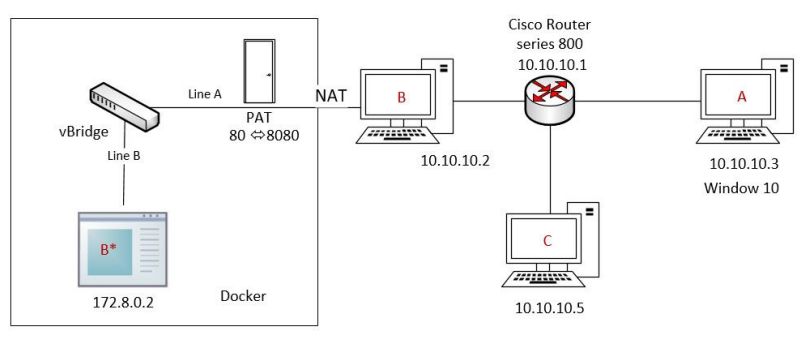

Fig. 5 Experimental model - Three physical computers as above connected by a router Cisco 800 Series Routers CISCO881-K9, computer $\mathrm{C}$ is attacker which can eavesdrop and inject packets into TCP connection between Host A and Hypervisor B. Docker container B* use Nginx for server with the local port is 80 and the public port is 8080 .

services was $0.6 \mathrm{Mbps}$. This result is much bigger than 120 bytes (two packets) sent by the attacker C. We keep experiment for 24 hours and it's still working. This proves that Son's hypothesis [7] is correct. The TCP connection is stuck in the CLOSE-WAIT state while the process established TCP connection keeps running. As the ACK-Storm activated by a couple of FIN-ACK packets can be last forever, the attacker could completely raise DDoS attack to hypervisor $\mathrm{B}$ and virtual machine $\mathrm{B}^{*}$. If an attacker could raise a DDoS attack with all available ports, about 65500 ports, he could "play" a DDoS attack which 94 million packets per second (with $40 \mathrm{Gbps}$ ), and also cause the port exhaustion.

In Line B: As explained above, in the ACK-Storm DoS attack experiment, only the first fake FIN-ACK packet, which we created, is forwarded to virtual machine $\mathrm{B} *$ through vSwicth Still, no retransmitted ACK packet is directed to virtual machine $\mathrm{B}^{*}$, the same result with the ACK-Storm DoS attack. We assume that vSwitch responses all retransmitted ACK packets instead of virtual machine $\mathrm{B}^{*}$ as long as no RST request is sent between Host $\mathrm{A}$ and virtual machine $\mathrm{B}^{*}$. To prove this, we try suspending virtual machine $\mathrm{B}^{*}$, the ACK-Storm attack still going on. We keep experiment for 24 hours more and the ACK-Storm attack is no sign of stopping. When we resume virtual machine $\mathrm{B}^{*}$, nothing happens. This is a feature of VMware workstation that prevent all invalid packets to virtual machine inside it.

\subsection{Experiment in Docker}

Hypervisor: We use Docker Desktop v2.2.0.5 with Docker Engine v19.03.8, Docker Compose v1.25.4. We build a new image depending on base image Ubuntu 18.04 and install Htop for monitoring the container's activity. We also use TCPdump for monitoring network flow, and use Nginx for server B.

\subsubsection{Original ACK-Storm DoS attack}

Attack Execution: With TCP connection created by using Chrome to access to the server Nginx in Container B* and

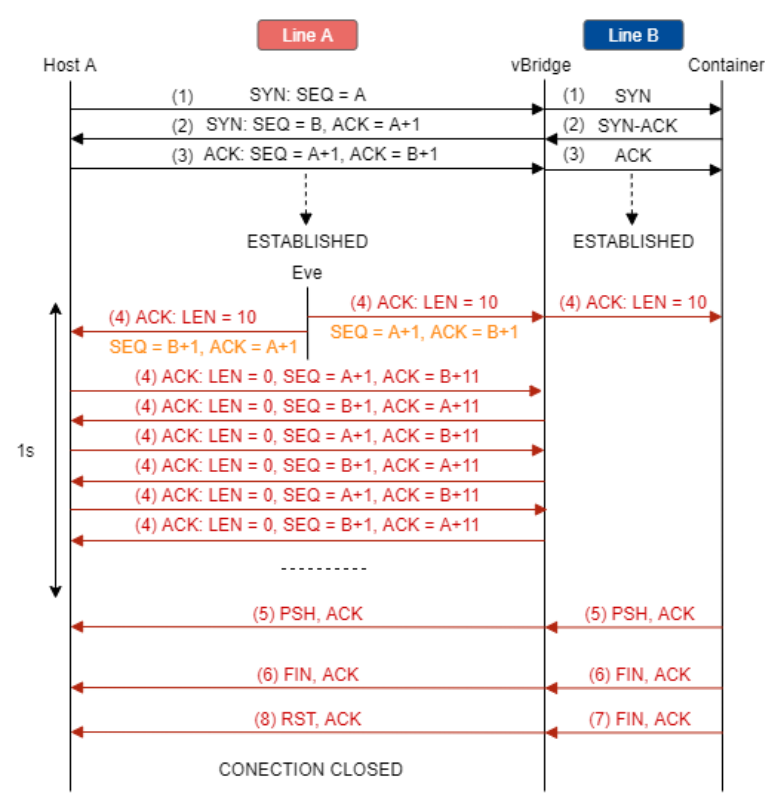

Fig. 6 TCP communication during the experiment in Docker with Ack packet

do the same as experiment original ACK-Storm DoS attack in VMware Workstation, we pick the last ACK packet in the TCP connection between Host A and Docker container B* for obtaining SEQ and ACK number. Then, we use scapy to create a couple of fake ACK packets with source IP is one party and destination IP is the other. After that, we send those ACK packets to each of the respective parties.

Analysis: When each party received a fake ACK packet, they responded with invalid retransmitted ACK packets, and the ACK-Storm was begun. The ACK-Storm was terminated by Host A (see Fig. 5) after 1 second while TCP connection was timeout after 30 seconds.

In Line A: We captured about 550 retransmitted ACK packets in 1 second while the ACK storm was occurring. 10 seconds later, Container B* send an RST-ACK packet. The TCP connection timeout after 30 seconds. There are still some retransmitted ACK packets generated by the original ACK-Storm attack but the retention time is very short. This is because of Docker's feature (or Nginx's) when the container received many retransmitted ACK packets with the same ACK/SEQ number. This result is not as expected but still proves a flaw in TCP connection, which was discovered by Abramov [6], when receiving not-yet-sent acknowledges data packet.

In Line B: The same result with the experiment ACKStorm DoS attack, only the first fake ACK packet is forwarded to Docker container $\mathrm{B}^{*}$ through vBridge. No retransmitted ACK packet is directed to Docker container B*. It seems like vBridge response all retransmitted ACK packets instead of Docker container B*, same behavior with vSwitch. 


\subsubsection{ACK-Storm DoS attack using FIN-ACK packet}

Attack Execution: We do the same with the experiment ACKStorm DoS attack, however we use a couple FIN-ACK packets instead of a couple of ACK packets. It means that we just turn the bit FIN flag to 1 and keep all conditions as the experiment ACK-Storm DoS attack as Section 3.3.1.

Analysis: When each party received fake FIN-ACK packets, they responded with invalid retransmitted ACK packets, and the ACK-Storm was begun.

In Line A: We captured a few retransmitted ACK packets between Host A and Container B* before Container B*'s side stopped responding.

In Line B: Same as above, in the experiment ACK-Storm DoS attack, only the first fake ACK packet is forwarded to Container $\mathrm{B}^{*}$ through vBridge. No retransmitted ACK packet is directed to Container $\mathrm{B}^{*}$.

Propose: We tried to replace simple ACK packets for attacking by HTTP packets. However, we got the same results as subsection 3.3.1 and 3.3.2. Because ACK-Storm DoS attack with FIN-ACK [7] packet seem does not work with TCP connection created by browsers like Chrome or Firefox and Nginx, we propose a new attack method based on it. In particularly, the attacker $\mathrm{C}$ creates his own fake TCP connection with server B, sends a fake FIN-ACK packet to server B after three-ways handshake completed. Then, constantly he sends fake retransmitted ACK packets of the same format as retransmitted ACK packets of ACK-Storm DoS attack. Server B, more specifically vBridge, will respond to them and stuck in CLOSE-WAIT state. With a simple TCP connection, the CLOSE-WAIT state has no timeout [12], so we assume server B may be stuck in CLOSE-WAIT state forever. The detail of this attack method is described in Section 3.3.3.

\subsubsection{ACK-Storm DoS attack using FIN-ACK packet and fake retransmitted ACK packets from attacker}

Attack Execution: We use scapy (version 2.4.3) to create a fake TCP connection with Container B* by Three Steps Handshake as follows:

1. Use scapy create a fake SYN packet manually with the destination is Container $\mathrm{B}^{*}$, the source is attacker $\mathrm{C}$, ACK number is "0", SEQ number is voluntary, and send to Container B*.

2. Capture response SYN/ACK packet from Container B* then create a fake ACK packet depending on the SYN/ACK packet (use ACK and SEQ number).

3. Send the fake ACK packet to Container B* and the TCP connection is established.

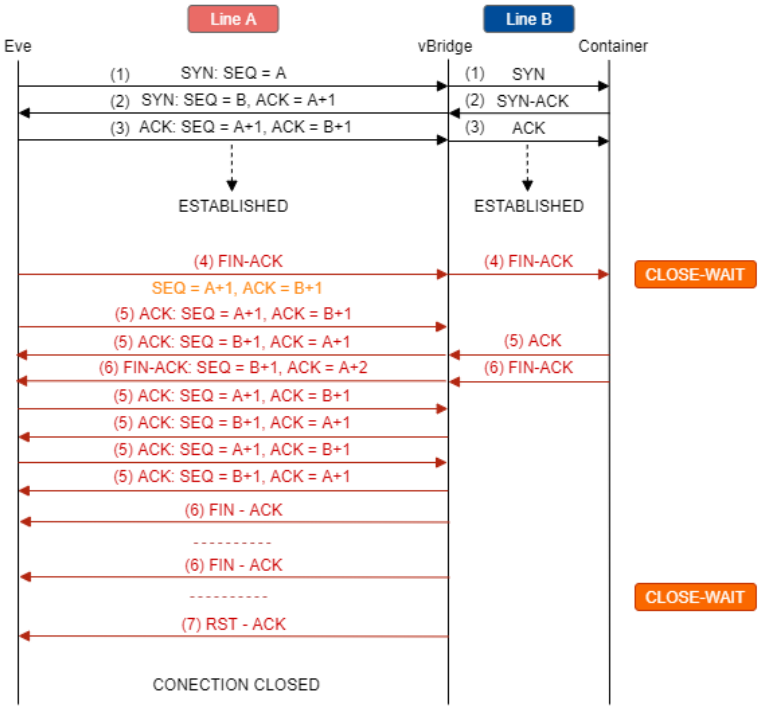

Fig. 7 TCP communication during the experiment using FIN-ACK packet in Docker with fake retransmitted ACK packets from attacker $\mathrm{C}$

After that, we create a fake FIN-ACK packet as same as the previous experiments and create a fake retransmitted ACK packets like attacker $\mathrm{C}$ received FIN-ACK packet similar to Container B*'s. Then, we follow these three steps:

1. Send the fake FIN-ACK packet to the Container B*.

2. Send the fake retransmitted ACK packet to the Container B*.

3. Delay about 1 second and repeat step 2 .

Analysis: When Container B* received the fake FIN-ACK packet, it switches to CLOSE-WAIT state and responses with invalid retransmitted ACK packets.

In Line A: Whenever vBridge receives the fake retransmitted ACK packet from the attacker $\mathrm{C}$, it responses with invalid retransmitted ACK packets. While the attacker $\mathrm{C}$ keeps sending fake retransmitted ACK packet to the Container B*, the Container B* is stuck in CLOSE-WAIT state. However, the attack does not last too long as same as the experiment ACK-Storm DoS attack with FIN-ACK packet [7]. After about 10 minutes, Container $\mathrm{B}^{*}$ sends an RST packet to close the TCP connection. In the experiment, we dump some FIN-PSH-ACK packets but there are no signs of disconnection until the Container B* sends RST packet suddenly. This might be a feature of vBridge when received too many retransmitted ACK packets with the same ACK/SEQ number.

In Line B: Only valid packets are directed to Container $\mathrm{B}^{*}$, the same as the result of the experiment ACK-Storm DoS attack. No retransmitted ACK packet is directed to Container $\mathrm{B}^{*}$. It means that vBridge response all retransmitted ACK packets instead of Container $\mathrm{B}^{*}$ as long as no RST request is sent between Host $\mathrm{A}$ and Container $\mathrm{B} *$. 


\subsection{Discussion}

\subsubsection{Feasibility}

From the results of the above experiments, we have concluded that the ACK-Storm DoS attack is possible with basic TCP connections in a virtualized environment. Based on the vulnerability discovered by Abramov [6] described in RFC 793 [12] and based on the idea of developing a method of attack with FIN-ACK packet of Son [7], an attacker can perform an attack which is made by creating a fake connection and sending packets just like a normal TCP connection being attacked by ACK-Storm DoS using FIN-ACK packet.

In our experiment, this attack method is feasible even with virtualization environments like Docker with Nginx for the server. However, in the default configuration of Nginx, each state of the connection has a timeout setting so the attack method using ACK packets is blocked and the risk of being attacked by an attacker with the FIN-ACK packet is also limited. However, attacker $\mathrm{C}$ can use a simple python script with scapy to create many connections to the server on the Docker container that uses Nginx to occupy ports within 10 minutes and can cause an issue, which is called port exhaustion, with vBridge when they combine with a botnet. The time, just about 10 minutes, is not too long, but the damage to businesses will not be small even though the cost of conducting the attack is not very high. With this attack method, attackers do not need to eavesdrop TCP connection but still can occupy port.

\subsubsection{Countermeasures}

As we mentioned above, in our attack experiments, the vSwitch (or the vBridge) responds (or ignores) invalid packets instead of the hypervisor (or the container) and only directs valid packets to the hypervisor. Therefore, the hypervisor will receive no packets from the client during the ACKStorm DoS attack. So, we can create a timer running on another thread to count the time unresponsive from the client and close that connection when "timeout". Besides, we can create a duplicate-ACK-checker to count the number of duplicate retransmitted ACK packets, when the number reaches the maximum, the hypervisor creates its own RST-ACK packet to force the connection stop completely.

\subsubsection{Ethical Considerations}

We disconnect the Internet when conducting our experiments and only tested attacks with the lab computers during the process. These computers only connected to each other during the experiment and did not connect even to the university local area network. Therefore, our experiments are completely harmless to the Internet.

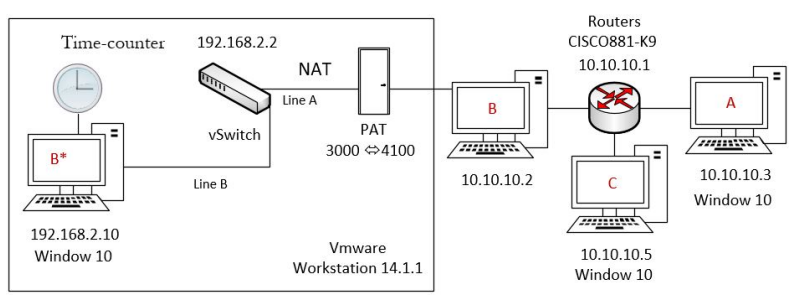

Fig. 8 Prevention ACK-Storm DoS attack model with Unresponsivetime counter

\section{Proposing solutions to prevent attacks on virtual systems}

4.1 Unresponsive-time counter

\subsubsection{Our proposed prevention model (see Fig. 8)}

Our proposing main idea is based on the results of the above experiments. On Line B of both VMWare and Docker experiments, only the first fake FIN-ACK/ACK packet, which we created, is forwarded to Virtual machine/Docker container $\mathrm{B}^{*}$ through vSwicth and no retransmitted ACK packet is directed to Virtual machine/Docker container B*, because vSwitch/vBridge responses all retransmitted ACK packets instead of Virtual machine/Docker container B*. This results in we could add a Unresponsive-time counter, which counts the time unresponsive from the client. We set a suitable timeout for each port/service. When the unresponsivetime counter reach the trigger value (timeout), it disconnects the corresponding connection.

\subsubsection{Mechanism}

Each service of virtual server will be designed with 3 threads A, B, C operating as follows:

1. Thread A will maintain the virtual server's primary service with IP_server:Port_server

2. Thread B, with a preset timeout value, is triggered when a service on stream A establishes a TCP connection, and is responsible for running a timer that does not receive a response, called unresponsive-time, to the corresponding port address. When unresponsive-time = timeout, thread B disconnects on corresponding TCP connection thread A.

3. Thread C listens for packets to IP_server:Port_server on thread A. If there is a correct incoming packet, reset unresponsive-time $=0$.

\subsubsection{Experimental results}

For testing the effectiveness of the solution, we integrate a module unresponsive-time counter in the python socket, 


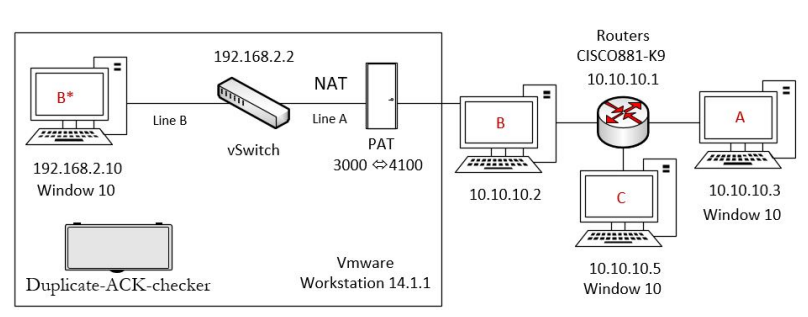

Fig. 9 Prevention ACK-Storm DoS attack model with DuplicateACK-packets counter

which is used to create connection in the above attack experiments. Then, we do the ACK-Storm DoS attack using FINACK packet experiment on virtual systems (VMware Workstation and Docker) as described. With the timeout value of the module unresponsive-time counter is set to 120 (seconds), the ACK-Storm was forced to terminated completely after 120 seconds from the start. This result proves that the prevention method using an unresponsive-time counter has a positive effect, overcoming the trapped in the CLOSEWAIT state, because of no timeout for that state, when a TCP connection is attacked by ACK-Storm DoS attack using FIN packet.

\subsection{Duplicate-ACK-packets counter}

\subsubsection{Proposed prevention model (see Fig. 9)}

Similarly, base on the results of the above experiments, all the retransmitted ACK packets have the same Acknowledgement number, we could use a small Duplicate-ACK-packets (DAP) counter running inside a host $\mathrm{A}$, which has virtual machine/docker container operating inside, to count number of ACK packets have the same Acknowledgement number. We set a maximum value for the DAP counter. When the counter reaches the maximum value, the counter creates a RST-ACK packet based on the retransmitted ACK packet to force the corresponding connection stop completely.

\subsubsection{Mechanism}

Because each port on the virtual server will provide a certain service, although each of these services can provide on many ports, we can use the value IP_server:Port_server as the key to store a value type "Counter", which is defined as below, in a dictionary. The type "Counter" use 6 properties for the key: [IP_host, Port_host, Flags, SEQ_number, ACK_number, LEN] to store the number of corresponding duplicate ACK packets:

1. IP_host is IP address of the host connecting with virtual server on corresponding IP_server:Port_server.

2. Port_host is Port address of the host connecting with virtual server on corresponding IP_server:Port_server.
3. Flags is on flags of packet.

4. SEQ_number is Sequence number of the packet.

5. ACK_number is Acknowledgement number of the packet.

6. LEN is the length of the packet.

\subsubsection{Experimental results}

The module duplicate-ACK-packets counter no need to be integrated in python socket (or to be integrated in the services of hypervisor), because it works as a standalone packet filtering program. We start duplicate-ACK-packets counter program before doing the ACK-Storm DoS attack using FINACK packet experiment on virtual systems (VMware Workstation and Docker). With the maximum value of DAP is set to 60 (packets), the ACK-Storm was forced to terminated completely after the module DAP couter receive 60 duplicate packets. This result proves that the prevention method using an duplicate-ACK-packets counter solve the problem of being stuck in the CLOSE-WAIT state, because of no timeout for CLOSE-WAIT state, when a TCP connection is attacked by ACK-Storm DoS attack using FIN packet.

\section{Conclusions}

In this paper, we have attempted to demonstrate the TCP ACK storm based DoS attack on vi0rtual and Docker networks. The attacked results have shown us the threats that can break down the virtual system.

For well-known software built by professional teams, such as Nginx, this type of attack is difficult to perform. However, its implications for the virtual server system are still evident once it is successfully implemented. This paper emphasizes the proper attention to handling timeout vulnerabilities with CLOSE-WAIT state in TCP connection when building and developing new software using TCP connection. This flaw could lead to an unlimited ACK-Storm DoS which could harm servers. Finally, TCP is still an important protocol and is used in many software, it was built long ago so inevitably there are vulnerabilities, so find the flaws and carefully study the vulnerabilities to find out methods to prevent and fix these gaps are the necessary work of all information security experts. In this paper, we have focused on researching and analyzing deeply the ACK-Storm DoS attack models of Abramov [6] and Son [7] as well as proposing a new attack version to capture the network ports of a virtual server. Based on the attacked results, we also have proposed two solutions for preventing the attacks on virtual and Docker networks: Unresponsive-time counter (see Section 4.1), and Duplicate-ACK-packets counter (see Section 4.2). Those had shown the efficiency to prevent the attacks that is implemented in our paper, and also had proved the assumption that $\mathrm{vSwitch} / \mathrm{vBridge}$ responses all retransmitted 
ACK packets instead of Virtual machine/Docker container are correct.

In the future, we will focus on researching other virtualized server systems such as Hyper-V, Oracle, and so on, with these types of attacks as well as proposing new attack measures and effective countermeasures.

\section{References}

1. J. McTigue, 2013 Virtualization Management Survey. (InformationWeek Reports, November 2012)

2. The 2020 State of Virtualization Technology. (Spiceworks reports, 2019), https://www.spiceworks.com/marketing/reports/stateof-virtualization. Accessed 18 April 2020.

3. Imperva's DDoS Attack reports, https://www.imperva.com/blog/this-ddos-attack -unleashed-the-most-packets-per-second-ever -heres-why-thats-important. Accessed 18 April 2020. https://www.imperva.com/blog/2019-globalddos-threat-landscape-report. Accessed 19 April 2020.

4. F. Bazargan, C. Y. Yeun, M. J. Zemerly, "State-of-theArt of Virtualization, its Security Threats and Deployment Models," International Journal for Information Security Research,Vol. 2, No. 3-4, pp. 335-343, 2012.

5. Hoxmeier, John A. and DiCesare, Chris, "System Response Time and User Satisfaction: An Experimental Study of Browser-based Applications" (2000). AMCIS 2000 Proceedings. 347.

6. R. Abramov, A. Herzberg, TCP ack storm DoS attacks, pp.12-27. (Proceedings of the 26th IFIP TC 11 International Information Security Conference, June 2011), https://www.researchgate.net/publication/225532285_ TCP_ack_storm_DoS_attacks. Accessed 20 April 2020.

7. Son N. D., M. Mimura, H. Tanaka, An Analysis of TCP ACK Storm DoS Attack on Virtual Network, pp. 288-293. (2019 19th International Symposium on Communications and Information Technologies (ISCIT), Ho Chi Minh City, Vietnam, 2019), https://ieeexplore.ieee.org/document/8905220. Accessed 20 April 2020.

8. Wireshark: Network protocol analyzer, Wireshark v3.2.3, https://wireshark.org/docs/manpages/wireshark.html. Accessed 20 April 2020.

9. VMWare: Workstation for Windows, VMWare Workstation Pro 14, https://www.vmware.com/products /workstation. Accessed 10 April 2020.

10. Docker: Docker desktop for Windows, Docker Nginx, https://www.docker.com. Accessed 20 April 2020.
11. A. Bansal and P. Goel, Simulation and Analysis of Network Address Translation (NAT) \& Port Address Translation (PAT) Techniques, pp.50-56. (Int. Journal of Engineering Research and Application, ISSN : 2248-9622, Vol. 7, Issue 7, Part 2, July 2017), http://www.ijera.com/papers/Vol7_issue7/Part2/I0707025056.pdf, Accessed 20 April 2020.

12. RFC 793 - TRANSMISSION CONTROL PROTOCOL, DARPA INTERNET PROGRAM, PROTOCOL SPECIFICATION, pp.72-73. (Sep 1981), https://tools.ietf.org/html/rfc793. Accessed 20 April 2020.

13. J. Chelladhurai, PR. Chelliah, SA. Kumar, Securing Docker Containers from Denial of Service (DoS) Attacks, pp. 856-859. (2016 IEEE International Conference on Services Computing (SCC), San Francisco, CA, 2016)

14. A. Blenk, A. Basta, M. Reisslein, W. Kellerer, Survey on network virtualization hypervisors for software defined networking, pp.655-685. (IEEE Communications Surveys and Tutorials, 2016)

15. E. Bauman, G. Ayoade, Z. Lin, A Survey on HypervisorBased Monitoring. (ACM Computing Surveys, 2015)

16. N.M.M.K. Chowdhury, R. Boutaba, A survey of network virtualization, pp.862-876. (Computer Networks, 2010)

17. A. Fischer, J.F. Botero, M.T. Beck, H. de Meer, X. Hesselbach, Virtual Network Embedding: A Survey (IEEE Communications Surveys \& Tutorials, 2013)

18. Arch Linux: Network bridge, https://wiki.archlinux.org /index.php/Network_bridge. Accessed 10 April 2020.

19. N. Varis, Anatomy of a Linux Bridge, pp.58. (Proceedings of Seminar on Network Protocols in Operating Systems, 2012), https://wiki.aalto.fi/download/attachments/70789083 /linux_bridging_final.pdf. Accessed 10 April 2020.

20. A. Mallika, A. Ahsanb, M. Md. Z. Shahadata, J. Tsou, Man-in-the-middle-attack: Understanding in simple words. (International Journal of Data and Network Science 3, 2019) https://www.researchgate.net/publication/330249434_Manin-the-middle-attack_Understanding_in_simple_words. Accessed 15 September 2020.

21. F. Callegati, W. Cerroni, M. Ramilli, Man-in-theMiddle Attack to the HTTPS Protocol. (IEEE Security Privacy, 7, 2009).

22. Y. Desmedt, Man-in-the-middle attack. (In Encyclopedia of cryptography and security, 2011) Springer, Boston, 